\title{
Research of the acoustic efficiency of chamber cells of ICE exhaust silencers
}

\author{
Olga M atasova*, Vladimir Tupov \\ B auman M oscow State Technical University, 105005, M oscow, Russia
}

\begin{abstract}
To reduce the exhaust noise of internal combustion engines, exhaust silencer containing chamber cells are widely used. Cell design largely determines its acoustic efficiency as a function of frequency. Therefore, in this work, a theoretical study of the most frequently used chamber noise-suppressing cells of various designs is carried out. Designs with high acoustic efficiency in a wide frequency range have been determined. The results of the study will allow the designer, using these structures, to synthesize highly efficient ICE exhaust silencers.
\end{abstract}

\section{Introduction}

The most powerful source of vehicle noise is its exhaust system. Therefore, a properly designed exhaust silencer provides a significant reduction in overall vehicle noise. To reduce the noise of vehicles, reactive silencers are most often used, in the design of which chamber noise-suppressing cells are widely used.

When calculating silencers, the matrix method is successfully used, which allows theoretically to accurately predict the acoustic efficiency of a silencer during its design without carrying out a large amount of experimental research [1 - 9]. This method is used in this work to determine the acoustic efficiency of the most used design variants of the chamber cell.

\section{Subject of research}

When developing reactive silencers, the most frequently used element is an expansion chamber with inlet and outlet pipes [5]. The study carried out based on the matrix method, which made it possible to study the noise-absorbing properties of several variants of the chamber cell design, presented in Fig. 1.

\footnotetext{
* Corresponding author: olgasanm@ rambler.ru
} 


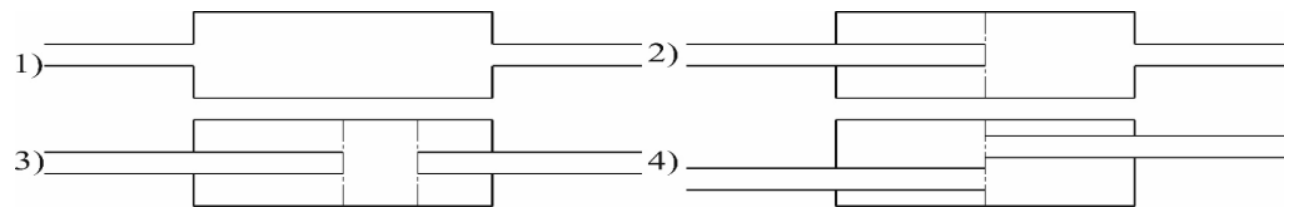

Fig. 1. Diagrams of constructive variants of the chamber cell.

Chamber cell 1 is a cylindrical chamber with inlet and outlet pipelines of the same flow section connected to it from the outside. In cell 2, the inlet pipeline is introduced to the middle of the chamber length. In cell 3 , the inlet pipeline is introduced to the middle of the chamber length, and the outlet pipeline is introduced to a quarter of its length. In cell 4, both pipelines are introduced to the middle of the chamber length.

Note that the matrix method calculation is applicable only in the framework of linear acoustics. In addition, this paper considers the plane-wave propagation, which is possible [3] to the boundary frequency:

$$
f_{b}=0,586 c / D_{c h}, H z
$$

where $c$ - speed of sound, $D_{c h}$ - inner chamber diameter.

\section{Calculation of the acoustic efficiency of the chamber cell}

The chamber cell, as applied to the exhaust silencers of the internal combustion engine, contains a gas flow moving in the direction of sound wave propagation from the source to the outlet. To simplify the consideration of the problem, let us assume that the pulsating flow is constant over the flow area of the cell and in time. To make it easier to take into account the moving gas flow when describing acoustic processes in a cell, we will use aeroacoustic instead of classical variables [1]:

$$
p_{c}=p(1+M)=p+M Z_{0} V ; \quad V_{c}=V(1+M)=V+M p / Z_{0}
$$

where $p$ and $V$ are sound pressure and mass vibrational velocity in the acoustic wave; $M$ is the $M$ ach number; $Z_{0}=c / S$ is characteristic impedance; $c$ is the speed of sound; $S$ - flow area.

The acoustic efficiency of a chamber cell can be described by the expression:

$$
\Delta L=10 \lg \left(W_{c, 1} / W_{c, 2}\right), d B,
$$

where $W_{c, 1}$ and $W_{c, 2}$ are the aeroacoustic power of sound waves, taking into account the flow of the medium, respectively, at the entrance and exit of the cell.

A eroacoustic power is calculated [1] by the formula:

$$
W_{c}=p_{c} V_{c} /(2 \rho),
$$

where $\rho$ is the density of the medium.

Substituting expression (4) into formula (3), taking into account the input and output, we obtain the dependence for calculating the acoustic efficiency of the cell in the form:

$$
\Delta L=10 \lg \left[p_{c, 1} V_{c, 1} \rho_{2} /\left(p_{c, 2} V_{c, 2} \rho_{1}\right)\right], d B .
$$

If, in the first approximation, the densities of the medium at the input and output of the cell are assumed to be the same, then formula (5) will be simplified:

$$
\Delta L=10 \lg \left[p_{c, 1} V_{c, 1} /\left(p_{c, 2} V_{c, 2}\right)\right], d B
$$


The aeroacoustic parameters of the wave at the input and output of the cell are related by the transmission matrix:

$$
\left[\begin{array}{l}
p_{c, 1} \\
V_{c, 1}
\end{array}\right]=\left[\begin{array}{ll}
A_{c} & B_{c} \\
C_{c} & D_{c}
\end{array}\right]\left[\begin{array}{l}
p_{c, 2} \\
V_{c, 2}
\end{array}\right]
$$

where $A_{c}, B_{c}, C_{c}, D_{c}$ are the aeroacoustic coefficients of the cell transfer matrix.

The cell transfer matrix can be obtained by multiplying the transfer matrices of the elements [4] forming this cell:

$$
\left[\begin{array}{ll}
A_{c} & B_{c} \\
C_{c} & D_{c}
\end{array}\right]=\left[\begin{array}{ll}
A_{c, 1} & B_{c, 1} \\
C_{c, 1} & D_{c, 1}
\end{array}\right]\left[\begin{array}{ll}
A_{c, 2} & B_{c, 2} \\
C_{c, 2} & D_{c, 2}
\end{array}\right] \cdots\left[\begin{array}{ll}
A_{c, n} & B_{c, n} \\
C_{c, n} & D_{c, n}
\end{array}\right]
$$

\section{Transfer matrixes of standard elements}

Chamber cells contain, as a rule, cylindrical pipelines of constant flow cross-section and a certain length, for example, inlet and outlet pipelines, a cell chamber, etc. elements. In fig. 2 shows such a section of a cylindrical pipeline of length $l$ and diameter $d$, where the arrow shows the direction of movement of the gas flow.

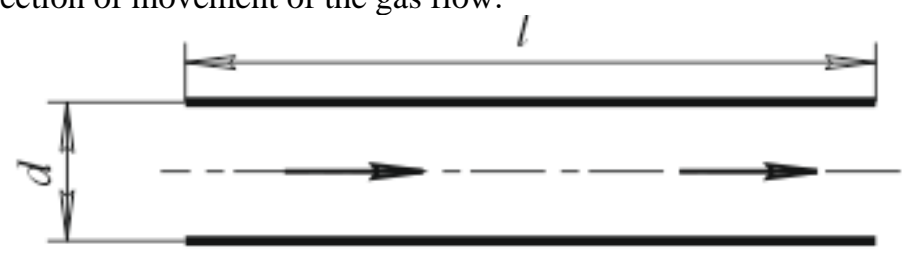

Fig. 2. Cylindrical pipeline.

The transfer matrix of such a cylindrical pipeline, taking into account the loss of acoustic energy and the movement of the medium [2], has the form:

$$
\left[\begin{array}{ll}
A_{c} & B_{c} \\
C_{c} & D_{c}
\end{array}\right]=\exp \left(-j k_{c} M l\right)\left[\begin{array}{cc}
\cos \left(k_{c} l\right)+\frac{\beta M}{k} \sin \left(k_{c} l\right) & j Z \sin \left(k_{c} l\right) \\
\left(\frac{j}{z}\right) \sin \left(k_{c} l\right) & \cos \left(k_{c} l\right)-\frac{\beta M}{k} \sin \left(k_{c} l\right)
\end{array}\right]
$$

where $Z=c(1-j \beta / k) / S$ - characteristic impedance of a pipeline; $S$-flow area; $k_{c}=$ $(k-j \beta) /\left(1-M^{2}\right) ; k=\omega / c$ - wave number; $\omega=2 \pi f ; f$ - acoustic frequency; $\beta$ - loss factor:

$$
\beta=\beta_{v}+\beta_{t}
$$

where $\beta_{v}$ is the loss coefficient associated with viscous friction and thermal conductivity of the medium; $\beta_{t}$ - associated with friction losses in turbulent flow.

We calculate these coefficients $[2,6]$ according to the following formulas:

$$
\begin{aligned}
& \beta_{v}=[P /(2 S c)] \sqrt{\pi f \mu / \rho}\left[1+(\gamma-1) \sqrt{\lambda /\left(C_{p} \mu\right)}\right] ; \\
& \beta_{t}=0,875 \psi M P /(2 S),
\end{aligned}
$$

where $\mu, \lambda$ and $C_{p}$ are the coefficients of dynamic viscosity, thermal conductivity and heat capacity at constant pressure of the exhaust gases; $P$ is the perimeter of the pipeline; $\gamma$ is the adiabatic index; $\psi$ - coefficient calculated by the formula:

$$
\psi=0,3164 / R e^{0,25} ;
$$

where $R e-$ Reynolds number in pipeline:

$$
R e=v d \rho / \mu,
$$


where $v$ - average velocity of movement of the medium in the pipeline.

Figure 3 shows the elements of a cell with abrupt expansion and narrowing of the flow area with pipelines inserted into the chamber ( $a$ and $b$ ) and with pipes attached to it from the outside (c and d).

a)

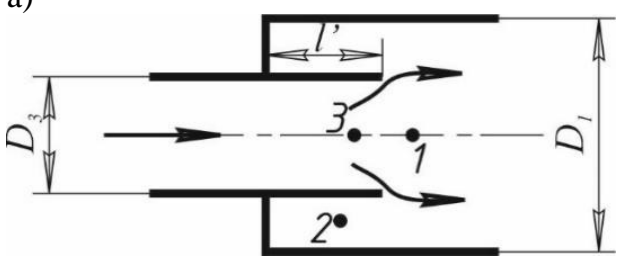

c)

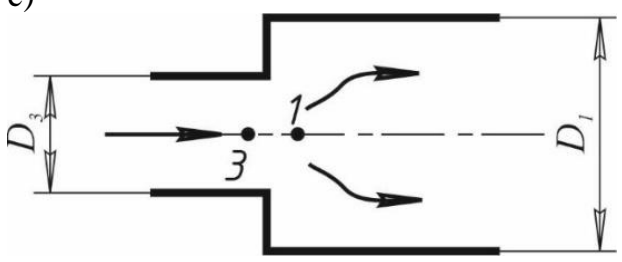

b)

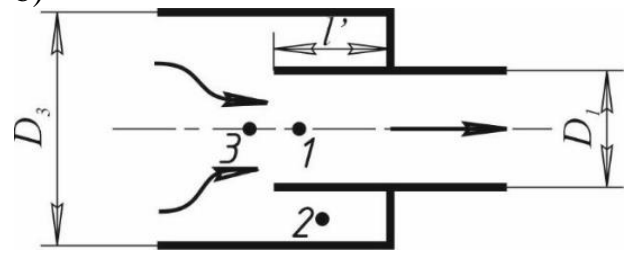

d)

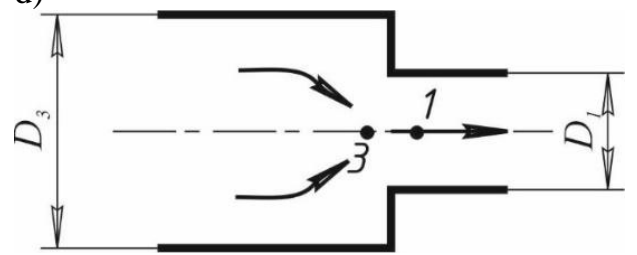

Fig. 3. Schemers of typical elements of a chamber cell: a) inlet pipeline introduced into the chamber; b) introduced outlet pipeline; c) abrupt expansion; d) abrupt narrowing.

For the input pipeline introduced into the chamber (Fig. 3a) and the output pipeline (Fig. $3 b)$, the transfer matrix coefficients are calculated [1] according to the following dependencies:

$$
\begin{aligned}
& A_{c}=1-K_{e x, n a r} M_{1}^{2} / E \\
& B_{c}=K_{e x, n a r} Z_{1} M_{1} / E \text {; } \\
& C_{c}=F^{-1}\left[-S_{3}\left(1-K_{e x, n a r} M_{1}^{2} / E\right)-C_{1} S_{1}\left(1-(\gamma-1) K_{e x, n a r} M_{1}^{4} / E\right)+\right. \\
& \left.+C_{2} S_{2} Z_{2}(\gamma-1) K_{e x, \text { nar }} M_{1}^{3} /\left(E Z_{1}\right)\right] \\
& D_{c}=F^{-1}\left[-S_{3}\left(Z_{1} K_{e x, \text { nar }} M_{1} / E\right)-\left(1-(\gamma-1) K_{e x, \text { nar }} M_{1}^{2} / E\right)\left(C_{1} S_{1} Z_{1} M_{1}-C_{2} S_{2} Z_{2}\right)\right] \text {, }
\end{aligned}
$$

where $C_{1}$ and $C_{2}$ are coefficients depending on the type of element (Table 1); $E=1-$ $M_{1}^{2} ; F=S_{3} Z_{3} M_{3}+C_{2} S_{2} Z_{2} ; K_{e x, n a r}$ - dynamic head loss coefficients for the case of expansion and narrowing of the gas flow, respectively. The indices for the $M$ ach number $M$, characteristic resistance $Z$, and cross-sectional area $S$ match the numbering of points in the elements in Fig. $3 ; Z_{2}$ is the input impedance of the dead-end part of the chamber with the flow area $S_{2}$, where the pipeline section is introduced into it to a depth of $l^{\prime}$ :

where

$$
Z_{2}=-j\left(c\left(1-j \beta_{v 2} / k\right) / S_{2}\right) c t g\left(k l^{\prime}\right),
$$

$$
\beta_{v 2}=\left[P_{2} /\left(2 c S_{2}\right)\right] \sqrt{\pi f \mu / \rho}\left[1+(\gamma-1) \sqrt{\lambda /\left(C_{p} \mu\right)}\right] ;
$$

here $P_{2}=P_{c h}+P_{p} ; P_{c h}$ and $P_{p}$ - the inner perimeter of the chamber and the outer perimeter of the pipeline introduced.

Table 1.

\begin{tabular}{|c|c|c|}
\hline Unit & $C_{1}$ & $C_{2}$ \\
\hline Introduced inlet pipeline & \multirow{2}{*}{-1} & +1 \\
\cline { 1 - 1 } Introduced outlet pipeline & & -1 \\
\hline
\end{tabular}


The dynamic pressure loss coefficients for a sudden expansion of the gas flow (Fig. 3a and 3c) $K_{e x}$ and with a sudden narrowing of the flow (Fig. 3b and 3d) $K_{n a r}$ is determined by the formulas:

$$
\begin{aligned}
& K_{e x}=\left(S_{c h} / S_{p}-1\right)^{2} ; \\
& K_{\text {nar }}=\left(1-S_{p} / S_{c h}\right) / 2,
\end{aligned}
$$

where $S_{c h}$ and $S_{p}$ are the flow area of the chamber and the pipeline, respectively.

For places of sudden expansion and contraction of the flow in the cell (Fig. 3c and 3d), the coefficients of the transfer matrices are calculated [1] by the formulas:

$$
\begin{aligned}
& A_{c}=1-K_{\text {ex,nar }} M_{1}^{2} / E ; \\
& B_{c}=K_{\text {ex,nar }} Z_{1} M_{1} / E ; \\
& C_{c}=(\gamma-1) K_{\text {ex, nar }} M_{1}^{3} /\left(E Z_{1}\right) ; \\
& D_{c}=1-(\gamma-1) K_{\text {ex,nar }} M_{1}^{2} / E .
\end{aligned}
$$

\section{Analysis of the results of studying chamber cells}

Calculation of chamber cells (Fig. 1) of an exhaust silencer with a chamber diameter of 80 $\mathrm{mm}$ and a length of $200 \mathrm{~mm}$ and a diameter of inlet and outlet pipelines of $20 \mathrm{~mm}$ was carried out under normal physical conditions using the $\mathrm{M}$ athcad 15 software product in the range of plane waves up to $2500 \mathrm{~Hz}$.

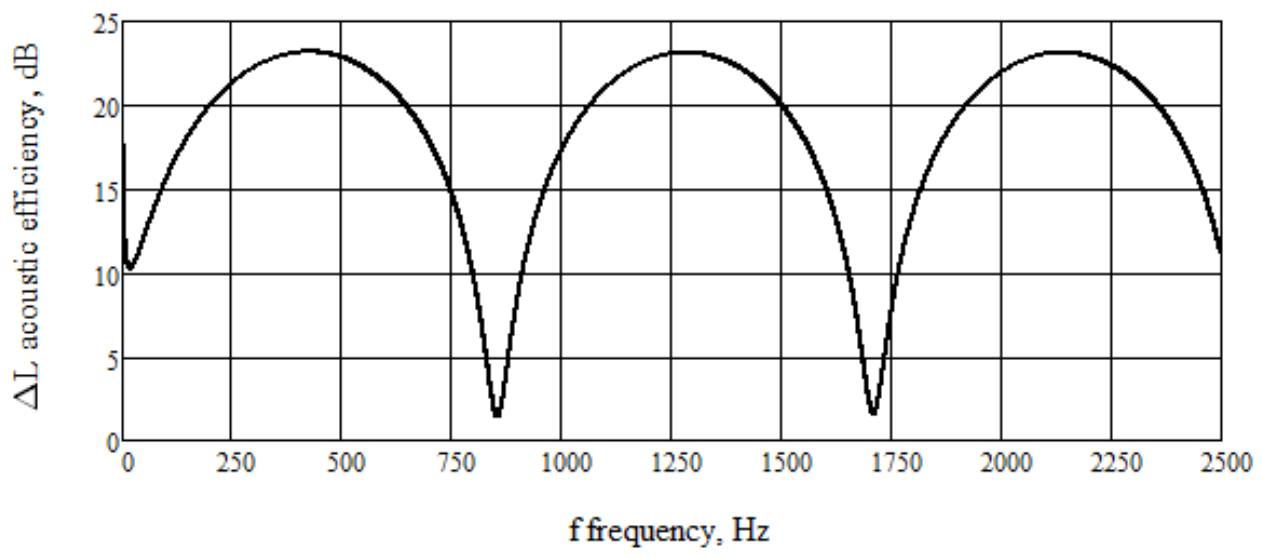

Fig. 4. Acoustic efficiency of cell 1 (Fig. 1) with losses and the flow $(M=0.05$ in the chamber).

Figure 4 shows the frequency dependence of the acoustic efficiency $(A E)$ of a chamber cell with external pipelines in the presence of a flow with $M=0,05$ in the chamber and losses of sound energy is cyclical, rising to $23 \mathrm{~dB}$ at the frequencies of longitudinal antiresonances of wave processes in chamber $428,75,1286$ and $2144 \mathrm{~Hz}$ and dropping to two decibels at resonance frequencies of 857.5 and $1715 \mathrm{~Hz}$, when an integer number of half-waves fits along the length of the chamber. At frequencies close to zero, the AE increases due to hydraulic losses in the places of abrupt change in the flow area when connecting pipelines to the chamber. 


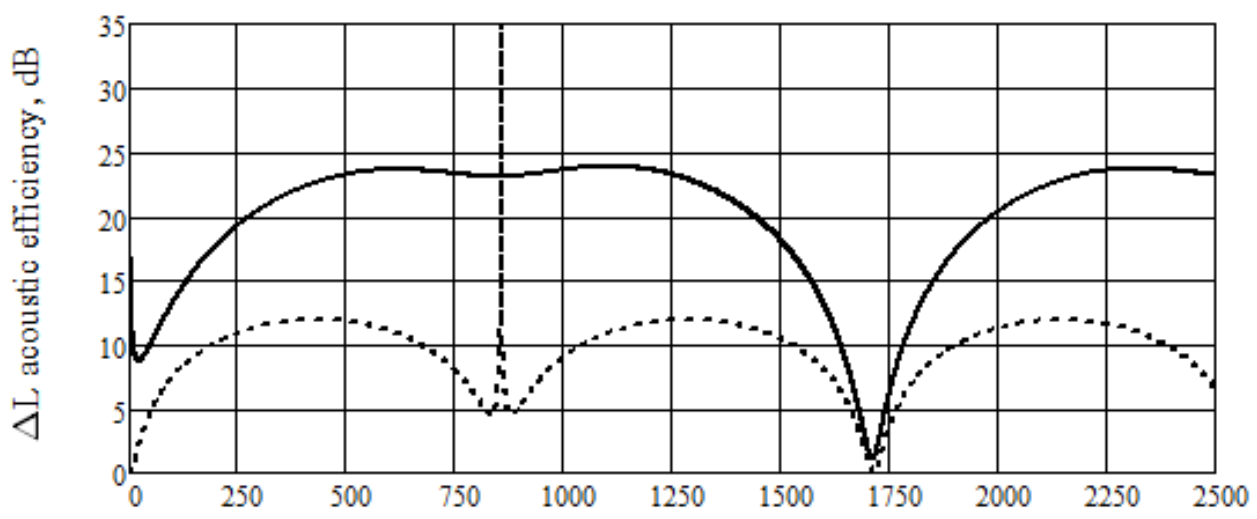

f frequency, $\mathrm{Hz}$

Fig. 5. The acoustic efficiency of cell 2 (Fig. 1) - . - - without the flow. with losses and flow $(M=0.05$ in the chamber);

Introduction of the inlet pipeline into the chamber until the middle of its length (Fig. 1), where the pressure at half-wave resonance at a frequency of $857.5 \mathrm{~Hz}$ is close to zero, leads to the phenomenon of almost complete reflection of waves from the outlet of the pipeline at this frequency, and the acoustic energy is almost not passes into the chamber and does not excite this natural frequency of the chamber. In addition, a quarter-wave resonator is formed between the outer wall of the pipeline and the inner wall of the chamber, tuned to the same frequency of $857.5 \mathrm{~Hz}$, at which its input impedance is close to zero, due to which the sound energy, closing on it, does not pass into the chamber and further into outlet pipeline. Figure 5 shows the diagram which is illustrates there is a peak similar increase in $A E$ at a frequency of $857.5 \mathrm{~Hz}$. A t the same time, at a frequency of $1715 \mathrm{~Hz}$, a longitudinal double half-wave resonance occurs in the chamber, as a result of which the pressure at the outlet of the introduced pipeline is no longer equal to zero, so the sound energy enters the chamber, amplifying the resonance process, and passes through it practically without attenuation, so as the distance between the holes of the pipelines is equal to half the length of this wave. The diagram shows a decrease in acoustic efficiency to zero at $1715 \mathrm{~Hz}$.

In the presence of an airflow with $M=0.05$, resistance arises in the chamber at the hole of the introduced pipeline, caused by losses during the abrupt flow expansion, therefore, there is no longer a significant reflection of sound energy from the opening of the pipeline, and it partially passes into the chamber and then into the outlet pipeline. In addition, part of it is spent on feeding the half-wave longitudinal resonance of the wave in the chamber, due to which there is a slight decrease in the cell efficiency at a frequency of $857.5 \mathrm{~Hz}$ (Fig. 5). At the same time, there is an increase in $A E$ at low frequencies due to hydraulic losses in places of abrupt enlargement and contraction of the air flow, as well as a slight increase $(\approx 1 \mathrm{~dB})$ of this characteristic at a frequency of $1715 \mathrm{~Hz}$, due to additional losses during a turbulent flow of the medium. 


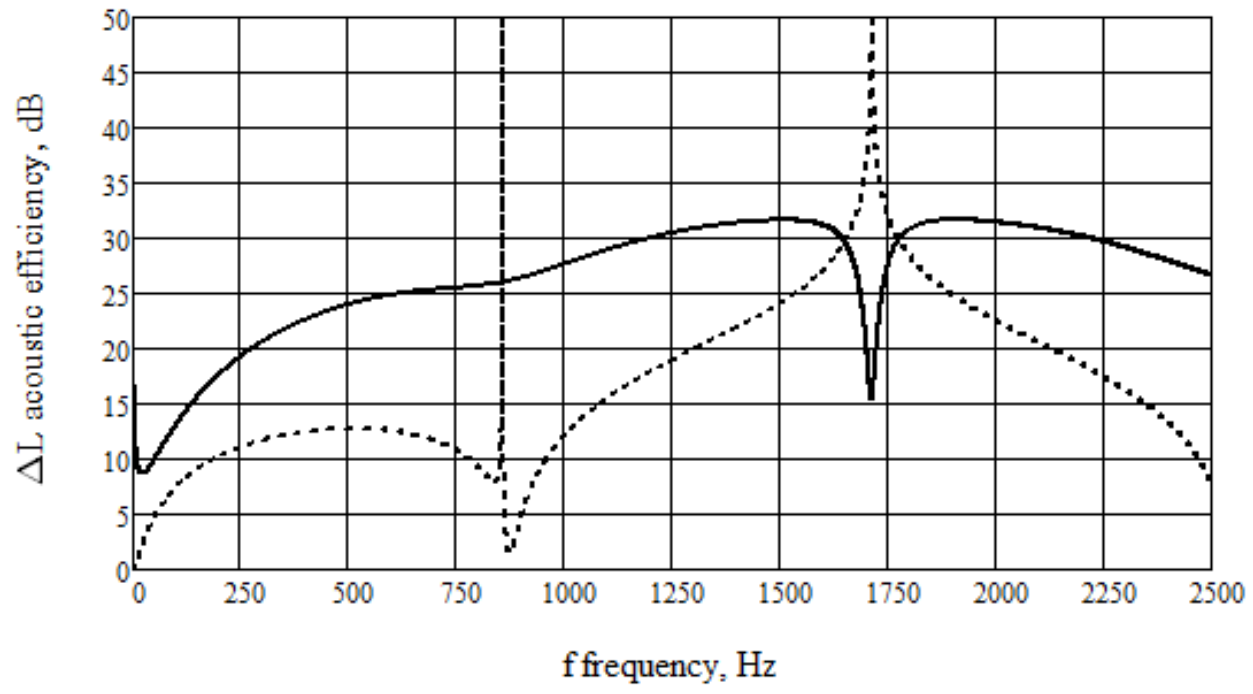

Fig. 6. The acoustic efficiency of cell 3 (Fig. 1) with losses and flow $(\mathrm{M}=0.05$ in the chamber); - - - - without the flow.

In order to eliminate an abrupt decrease in the acoustic efficiency at a frequency of 1715 $\mathrm{Hz}$ due to a double half-wave longitudinal resonance of the medium in the chamber, an outlet pipeline was introduced into its cavity to a depth equal to a quarter of its length (Fig. 1). Then, a quarter-wave resonator is formed between the walls of the pipeline and the chamber, the input impedance of which is close to zero at a frequency of $1715 \mathrm{~Hz}$, and the main part of the acoustic energy is closed to it, without passing into the output pipeline. At the same time, at the pipeline hole, the pressure at this frequency in the chamber is close to zero; therefore, only an insignificant part of the acoustic energy arrives at the cell output, as a result of which a peak similar to an increase in the AE is observed at this frequency (Fig. 6).

When an air flow with $M=0.05$ is imposed in the chamber, hydraulic losses arise in the inlet of the outlet pipeline, and acoustic vibrations, amplified by the double half-wave resonance in the chamber, pass to the cell outlet, abruptly reducing the acoustic efficiency at a frequency of $1715 \mathrm{~Hz}$ (Fig. 6). A t low frequencies, a slight increase in $\mathrm{AE}$ is observed due to hydraulic losses during expansion and contraction of the flow. 


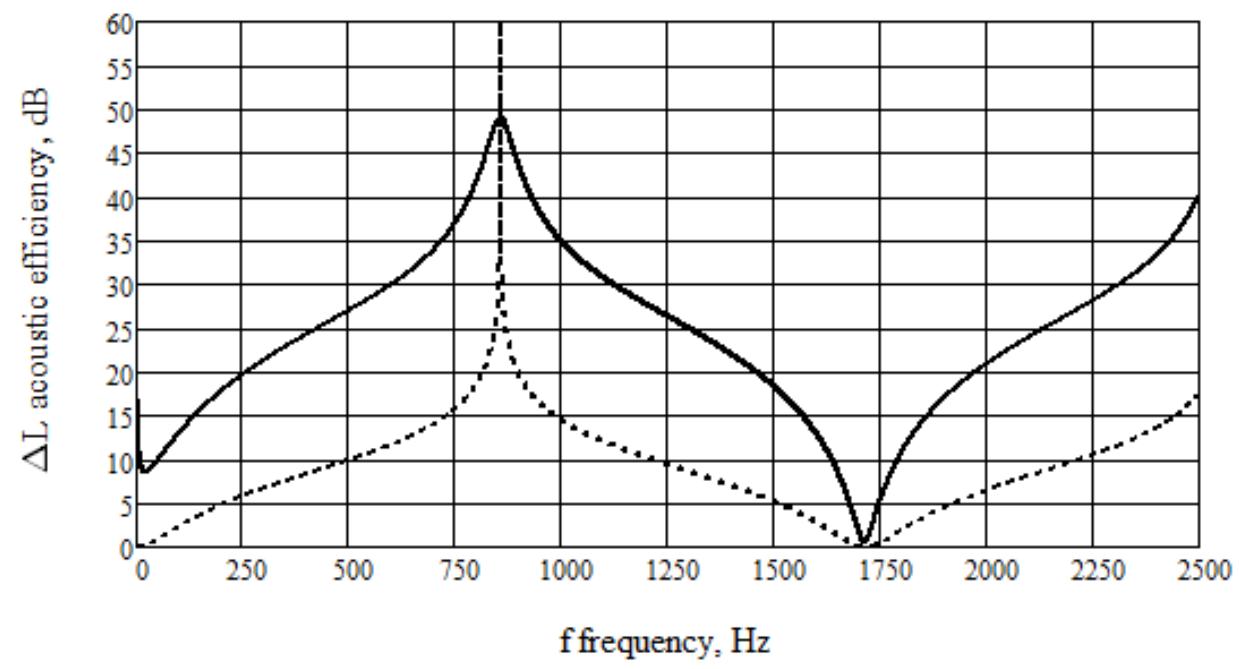

Fig. 7. The acoustic efficiency of cell 4 (Fig. 1) with losses and flow $(\mathrm{M}=0.05$ in the chamber $)$; - - - without the flow.

For a more efficient suppression of half-wave resonance in the chamber, both pipelines were introduced to the middle of its length at different levels (Fig. 1). B etween the walls of the pipelines and the chamber, two quarter-wave resonators were formed, the input total impedance of which is half the impedance of each of them separately and dropped to almost zero at a frequency of $857.5 \mathrm{~Hz}$. In addition, the location of the pipeline openings in the zone of zero pressure in the chamber at this frequency minimizes the passage of acoustic energy to the cell output and causes a peak similar rise in AE (Fig. 7). A t a frequency of $1715 \mathrm{~Hz}$, the input impedance of the formed quarter-wave resonators tends to infinity, since their length al ready corresponds to half the length of this wave, and they almost completely reflect the acoustic energy that passes to the cell output without attenuation. A this frequency, zero $A E$ value is observed on the diagram.

The introduction of an air flow with $M=0.05$ into the chamber leads to the appearance of hydraulic losses in the pipeline openings, due to which their end impedance is no longer close to zero, and the acoustic energy, amplified by the half-wave resonance of the chamber, partially passes to the cell output at a frequency of $857.5 \mathrm{~Hz}$, lowering the $A E$ to $49 \mathrm{~dB}$ and expanding the near-resonant area of sound damping. A the same time, an increase in acoustic efficiency is observed at low frequencies and insignificantly at a frequency of $1715 \mathrm{~Hz}$ due to hydraulic and turbulent losses in the cell (Fig. 7).

The analysis of the results of the conducted studies of the acoustic efficiency of the considered chamber cell designs shows that the last two cell designs with pipelines introduced into the chamber have a relatively high acoustic efficiency in a wide frequency range when a gas flow is imposed, which makes it possible to recommend them for use in exhaust noise silencers of internal combustion engines.

\section{Conclusion}

$B$ ased on the research, the influence of various designs of the chamber cell of exhaust silencer its acoustic efficiency depending on the sound frequency has been studied. It is shown that the introduction of the inlet and outlet pipelines into the chamber at half of its length, as well as the simultaneous introduction of the inlet pipeline by half and the outlet pipe at a quarter of its length, allows one to obtain high values of the acoustic efficiency of the chamber cell 
in a wide frequency range. Thus, the obtained results of the study will allow the designer to synthesize chamber cells with the required acoustic efficiency in a given frequency range, and can be used in the development of exhaust silencers for internal combustion engines.

\section{References}

1. M.L. M unjal, Acoustics of Ducts and Silencers. - N.Y .: W ileyentersscience, 5 - 320, (1987)

2. A.I. Komkin, V.V. Tupov, On the calculation of acoustic characteristics of silencers, Herald of the BM STU. Series M echanical Engineering. 3, 118 - 124 (1994)

3. E. Skudrzyk, The Foundations of Acoustics. Basic Mathematics and Basic Acoustics. Springer-V erlag. W ien, N ew Y ork, 25 - 915, (1971)

4. V.A. Kulkina, V.V. Tupov, Assessment of the acoustic efficiency of reactive silencers of vehicles' noise. Overview. A coustics of the environment: of the Third All-Russian conference of young scientists and specialists' proceedings. Bauman Moscow State Technical University, 144 - 147 (2018)

5. V.M. Kondrashov, Y u.S. Grigoriev, V.V. Tupov and other. Two-stroke carburetor internal combustion engines, - M .: ed. M echanical engineering, 215 - 270, (1990)

6. N.A. Dubskaya, V.V. Tupov, Calculation of the acoustic efficiency of the intake silencer of a small-size internal combustion engine. A coustics of the environment: of the Third All-Russian conference of young scientists and specialists' proceedings. Bauman M oscow State Technical U niversity, 72 - 77, (2018)

7. V.V. Tupov, The Calculation of Channel End Hole Reflection Ratio Without a Flange, in proceedings of ISP Conference "Modeling in Education 2019", (2019)

8. A.I. Komkin, A.I. Bykov, M .A. M ironov Acoustic impedance of the hole at high sound pressure levels. A coustical Physics, 64(5), 562 - 565, (2018)

9. A.I. Komkin, A.I. Bykov, M.A. M ironov Inertial attached orifice length at high sound pressure levels. A coustical Physics, 64(3), 296 - 301, (2018) 